\title{
TENDENCIAS DE CAMBIO DE ESPECIES FORESTALES EN PLANTACIONES DE PEQUEÑOS Y MEDIANOS PROPIETARIOS
}

\author{
Ávila, Alberto ${ }^{5}$ y Muñoz, Juan Carlos ${ }^{1}$
}

\section{RESUMEN}

El Instituto Forestal (INFOR), dentro de su Área de Investigación Inventario y Monitoreo de los Ecosistemas Forestales y su Línea de Investigación Inventario Forestal Continuo, actualiza anualmente las superficies de plantaciones entre las regiones de Coquimbo y Aysén y, con una frecuencia menor ( 3 a 4 años) y en forma rotativa, las existencias volumétricas por regiones, tanto de bosques nativos como de plantaciones. Complementariamente, realiza proyecciones periódicas de la disponibilidad futura de madera en las plantaciones para períodos futuros.

Las especies principales en las plantaciones forestales del país son pino radiata (Pinus radiata) y eucaliptos (Eucalyptus globulus y E. nitens), que en conjunto representan el $93 \%$ de los 2,4 millones de hectáreas de bosques plantados a diciembre de 2012 , correspondiendo el $7 \%$ restante a varias otras especies, principalmente de los géneros Acacia, Eucalyptus, Pseudotsuga, Pinus, y Populus. Pino radiata fue por años la especie preponderante en las plantaciones forestales. Se estima que a principios de los años 70 existían en el país unas 450 mil hectáreas de plantaciones forestales y casi el $90 \%$ de esa superficie correspondía a esta especie.

Debido a las extensas superficies de suelos forestales desarbolados disponibles para la forestación en el país y en gran parte como resultado de los incentivos del Estado para esta actividad, las plantaciones se incrementan posteriormente en forma progresiva, pero el nivel de participación de pino radiata en ellas se mantiene relativamente constante hasta principios de los años 90 cuando la participación de eucaliptos se hace creciente y de 102 mil hectáreas en 1990 sube a 302 mil hectáreas en 1995, a 552 mil hectáreas en 2005 y a 774 mil hectáreas en 2012.

A diciembre de 2012 las plantaciones en el país alcanzan a 2,4 millones de hectáreas y esta superficie está compuesta en un $61 \%$ por pino radiata, $32 \%$ por eucalipto y el $7 \%$ restante por otras especies. Este creciente reemplazo de pino radiata por eucalipto en las plantaciones se debe en gran medida a la información proporcionada por las diferentes líneas de investigación sobre eucalipto que INFOR ha desarrollado desde los años 60 del siglo pasado, que han abordado desde la introducción y selección de especies, las técnicas de producción de plantas, las técnicas de establecimiento de plantaciones, los estudios de crecimiento y manejo silvícola, y las propiedades físicas y mecánicas de la madera, hasta los más recientes programas de mejoramiento genético que INFOR mantiene en colaboración con las principales empresas forestales del país.

El presente trabajo se desarrolló como apoyo al estudio Proyección de la Disponibilidad de Madera de Plantaciones de Pino Radiata y Eucalipto (2010-2040) (INFOR, 2013), con el objeto de sustentar los necesarios supuestos de la proyección en materia de superficies de plantaciones y composición de especies de estas para el periodo de proyección. El trabajo se realizó determinando índices de tendencias en el cambio de especies por regiones, sobre la base de las series de superficie de plantaciones acumuladas por especie y región en el período 1991-2010 y de información similar en lo referente a reforestación por especie y región para el período 2006-2011, en ambos casos para el segmento de pequeños y medianos propietarios.

Palabras clave: Eucalyptus spp, Pinus radiata, Superficies Plantaciones Forestales.

\footnotetext{
${ }^{5}$ Ingenieros Forestales, Instituto Forestal, investigadores Sede Bío Bío, Concepción aavila@infor.cl; jmunoz@infor.cl
} 


\section{SUMMARY}

The Chilean Forestry Institute (INFOR), under the framework of its Forest Ecosystems Continuous Inventory Program, updates each year the planted forest area in the country between the Coquimbo and Aysén regions and, with a lower frequency ( 3 - 4 years) and in a rotating way, updates also the stocking by region, both for planted and native forests. Additionally INFOR carries out periodic wood availability projections in planted forests involving 30 year projection scenarios.

Main species in Chilean planted forests are Radiata Pine (Pinus radiata) and Eucalypts (Eucalyptus globulus and E. nitens), species which represents $93 \%$ of the 2.4 million hectares total planted forests area in the country in 2012, the rest of the planted area corresponds to other species, mainly from the Acacia, Eucalyptus, Pseudotsuga, Pinus and Populus genus. Radiata Pine was for a long time the main species in planted forests. By the beginning of the 70s of the past century there were some 450 thousand hectares of planted forest in the country being in that time almost $90 \%$ of the area Radiata Pine plantations.

Because of the large areas of uncovered forest soils available to afforestation in the country and as a result of the State incentives to this activity, planted forest area increases later, but the Radiata Pine participation remains fairly constant until the early 90s when the Eucalypts participation began to increase; 102 thousand hectares in 1990, 302 thousand hectares in 1995 and 774 thousand hectares in 2012.

By the end of 2012 planted forests area in the country reached 2.4 million hectares and this area is composed by Radiata Pine (61\%), Eucalypts (32\%) and other species (7\%).

The increasing species replacement in planted forests has been greatly promoted as well by the results from different INFOR's research lines on Eucalypts, carried out since the 60s, facing species introduction and selection, nursery techniques, plantation establishment techniques, silviculture and management, genetic improvement, wood mechanical and chemical properties, utilization and others.

The present study has been developed as a support to the Wood Availability Projection in Planted Forests 2010 - 2040 study, carried out by INFOR in order to provide the necessary information to support the projection assumptions on planted areas and species composition.

Species replacement trend indexes by region were developed over the basis of plantation area series for the 1991-2010 period and similar information on reforestation by species and region for the period 2006-2011, in both cases considering the small and medium owners segment.

Key words: Eucalyptus spp, Pinus radiata, Planted Forest Areas. 


\section{INTRODUCCIÓN}

Debido a las extensas áreas de suelos disponibles para forestación, la necesidad de proteger esos suelos contra la erosión y el comparativamente lento crecimiento de las especies forestales nativas, el Instituto Forestal inició a principios de los años 60 del siglo pasado un amplio y sistemático programa de introducción de especies forestales al país.

Mediante este programa, que incluyó tanto coníferas como latifoliadas, fueron establecidas más de 8 mil parcelas experimentales entre las regiones de Coquimbo y Aysén, ensayos que ya en la década de los 70 empezaron a generar abundante información sobre el comportamiento y adaptación de diferentes especies de rápido crecimiento a través de las diferentes regiones del país.

Tempranamente en este programa de investigación destacaron diversas especies del género Eucalyptus y con las principales de ellas INFOR inició investigaciones complementarias en torno a su silvicultura y manejo; manejo de semillas, técnicas de producción de plantas y establecimiento de plantaciones y otras, para posteriormente iniciar programas de mejoramiento genético que se han intensificado hasta ahora. Toda esta información condujo a que, paulatinamente al principio y aceleradamente después, algunas de estas especies fueran incorporadas en los programas de forestación en el país.

Posteriormente, desde fines de los años 90, INFOR mantiene una línea de trabajo permanente de monitoreo de los recursos forestales representado por el Inventario Forestal Continuo de los Ecosistemas Forestales. Dentro de esta línea de trabajo, actualiza anualmente la superficie de plantaciones forestales, según las regiones administrativas del país y las especies que las componen, y cada tres a cuatro años las existencias madereras contenidas en ellas, y alg 0 similar efectúa con los bosques nativos según regiones y tipos forestales.

Las actualizaciones de las plantaciones registran que en 1980 existía un total plantado de 794 mil hectáreas, superficie de la cual el 90,2 \% correspondía a pino radiata (Pinus radiata), solo el $4,2 \%$ a eucaliptos (fundamentalmente Eucalyptus globulus, 33 mil hectáreas) y $5,5 \%$ a otras especies como álamos, pino oregón y otras. Tres décadas después, en 2012, la superficie de plantaciones ha aumentado a 2,4 millones de hectáreas y la participación de eucaliptos ha aumentado a 33\%, unas 792 mil hectáreas, con la presencia de dos especies principales (Eucalyptus globulus, 542 mil hectáreas, y Eucalyptus nitens, 232 mil hectáreas) y de varias otras de participación aún marginal (unas 18 mil hectáreas), como E. camaldulensis, E, regnans, E. delegatensis, E. viminalis y otras.

Con una periodicidad variable INFOR efectúa también una proyección de la disponibilidad de madera a futuro en las plantaciones forestales, según especie y región del país (INFOR, 2005, 2007), la última de ellas para el período 2010 - 2040 (INFOR, 2013).

Tanto en materia de las actualizaciones de superficies de plantaciones como en lo referente a las proyecciones de existencias madereras en ellas, INFOR mantiene un convenio con las principales empresas forestales, gracias al cual estas entregan su propia información, permitiendo así que INFOR concentre su trabajo y sus recursos en la obtención de la información correspondiente al segmento de pequeños y medianos propietarios forestales (PYMP).

Variable fundamental en la proyección de la disponibilidad de madera en el tiempo es la superficie plantada y las especies que la componen, base para una buena estimación de cómo evolucionará esta, por especie y en las distintas regiones. Dada la creciente participación de eucaliptos en las plantaciones y considerando el interés que ha despertado en el segmento de PYMP la alternativa de estas especies, de rotación más corta y con un mercado en ampliación como es el de la pulpa, el presente trabajo fue desarrollado como el apoyo necesario para la proyección 2010-2040 (INFOR, 2013), respecto de la evolución de la superficie de plantaciones en 
manos del segmento mencionado y de la tendencia de cambio de especies en este. El trabajo considera las tres especies principales Pinus radiata, Eucalyptus globulus y Eucalyptus nitens y abarca las regiones de O'Higgins a Los Lagos.

\section{OBJETIVOS}

Determinar la tendencia en el cambio de especies en las plantaciones forestales de pequeños y medianos propietarios forestales con el fin de respaldar los supuestos necesarios para las proyecciones a futuro de la disponibilidad de madera de plantaciones de pino radiata y eucaliptos.

\section{ANTECEDENTES}

\section{Evolución de las Plantaciones Forestales Nacionales}

Hasta principios de los años 90 del siglo pasado pino radiata predominaba fuertemente en las plantaciones forestales del país, representando en 1985 el $87 \%$ de la superficie total de estas. Posteriormente, la información entregada por INFOR sobre crecimiento y silvicultura de especies del género Eucalyptus propicia que a mediados de los 90 empiecen a extenderse las plantaciones con ellas, principalmente de Eucalyptus globulus, debido a su rápido crecimiento y la posibilidad de una corta rotación, a su vigorosa regeneración vegetativa que permite rotaciones sucesivas en monte bajo sin necesidad de plantar nuevamente y al precio de la madera pulpable, aspectos que la hicieron muy atractiva para el segmento de pequeños y medianos propietarios (PYMP), quienes en muchos casos comenzaron a reemplazar sus rodales de pino por esta especie. Se incorpora en las plantaciones en forma también creciente Eucalyptus nitens, que si bien no tiene una regeneración vegetativa tan vigorosa como para asegurar un monte bajo y su madera para pulpa tiene un valor algo menor que la primera, es más resistente a frío y su crecimiento es mayor.

La superficie de plantaciones de eucaliptos existente en 1985 (algo más de 50 mil hectáreas) se duplica para 1990, se sextuplica en 1995 y en 2012 llega ya a 774 mil hectáreas (Cuadro $\mathrm{N}^{\circ} 1$ y Figura $\mathrm{N}^{\circ} 1$ ), aumentando su participación un $4,3 \%$ a un $32 \%$. El total de plantaciones forestales en tanto se ha más que duplicado en el mismo período.

Cuadro $\mathrm{N}^{\circ} 1$

EVOLUCIÓN SUPERFICIE DE PLANTACIONES FORESTALES 1973 - 2012

\begin{tabular}{|c|c|c|c|c|}
\hline \multirow{2}{*}{ Año } & Pino radiata & Eucalipto & Otras & Total \\
\hline & \multicolumn{4}{|c|}{ ( M ha ) } \\
\hline 1973 & 400 & & 50 & 450 \\
\hline 1980 & 717 & 33 & 44 & 794 \\
\hline 1983 & 968 & 40 & 60 & 1.068 \\
\hline 1985 & 1.040 & 51 & 97 & 1.188 \\
\hline 1990 & 1.243 & 102 & 116 & 1.461 \\
\hline 1995 & 1.380 & 302 & 136 & 1.818 \\
\hline 2000 & 1.475 & 359 & 156 & 1.990 \\
\hline 2005 & 1.419 & 552 & 154 & 2.125 \\
\hline 2010 & 1.472 & 696 & 174 & 2.342 \\
\hline 2011 & 1.481 & 740 & 174 & 2.395 \\
\hline 2012 & 1.471 & 774 & 169 & 2.414 \\
\hline
\end{tabular}

(Fuente: INFOR, 2014)

Existen unas 18.000 ha adicionales de otras especies del género Eucalyptus que en la actualización de plantaciones están incluidas en la categoría Otras. 


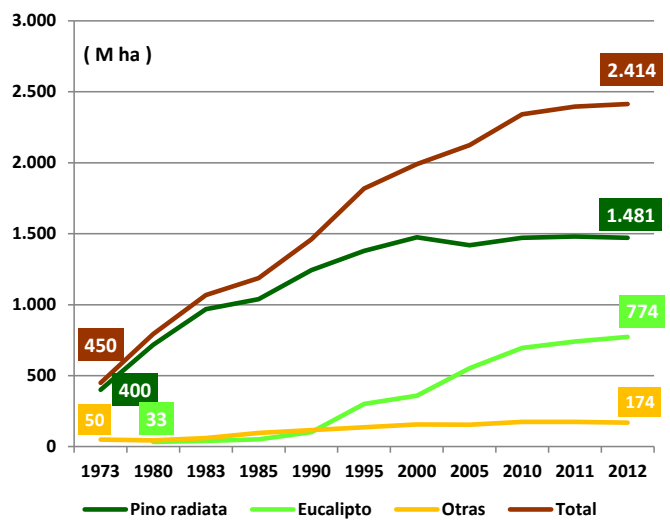

Figura $\mathrm{N}^{\circ} 1$

EVOLUCIÓN DE LA SUPERFICIE DE PLANTACIONES SEGÚN ESPECIES PRINCIPALES

\section{Evolución de las Plantaciones Forestales del Segmento PMYP}

INFOR define como pequeño propietario forestal a las personas, naturales o jurídicas, cuyo patrimonio total de plantaciones es inferior a 200 ha y como mediano propietario a aquellas con un patrimonio de plantaciones mayor o igual a 200 ha. No se incluye a las grandes empresas del sector. La superficie de plantaciones del segmento PYMP ha venido aumentando desde los años 90 del siglo pasado y desde inicios del presente siglo el incremento es ya bastante importante y llega en 2010 a una cifra cercana a las 750 mil hectáreas lo que representa en ese año ya el $32 \%$ aproximadamente de las plantaciones totales del país (Figura $\mathrm{N}^{\circ} 2$ ).

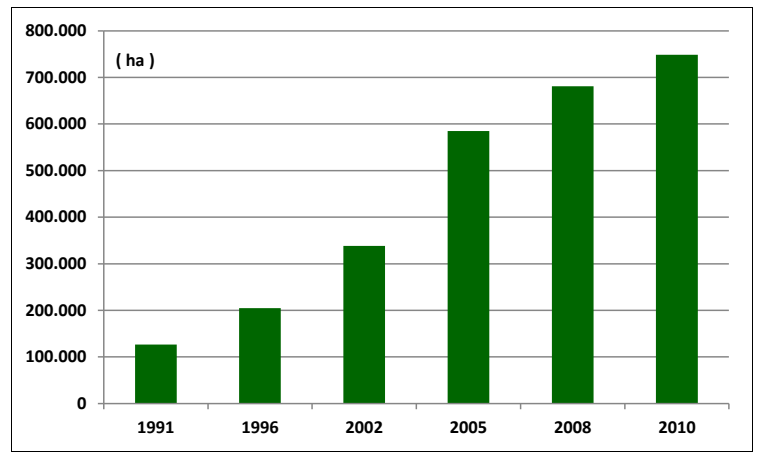

Figura $\mathrm{N}^{\circ} 2$

SUPERFICIE TOTAL PLANTACIONES PYMP POR AÑO

Las plantaciones forestales del segmento PYMP se distribuyen a través de todas las regiones incluidas en el estudio, se concentran principalmente entre las regiones de Maule y Araucanía (59,3\%) (Figura № 3), y entre los años 2004 y 2010 se han incrementado en unas 240 mil hectáreas $(47 \%)$. 


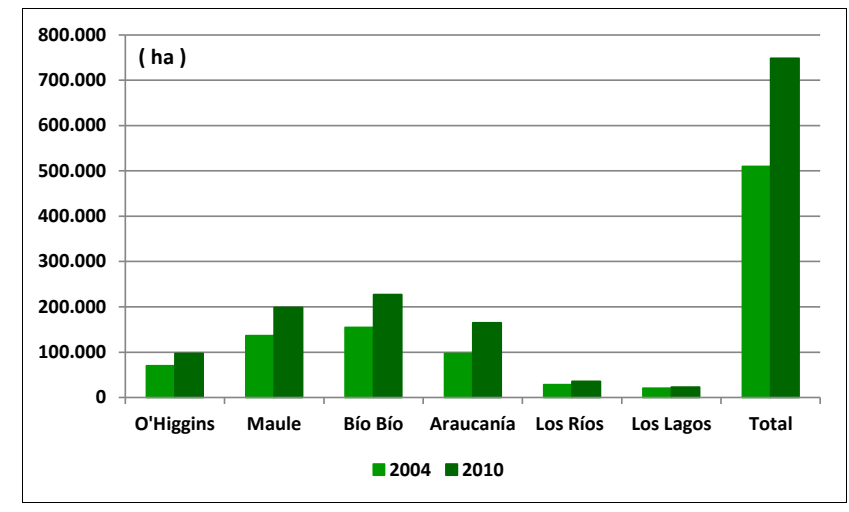

Figura $\mathbf{N}^{\circ} 3$

SUPERFICIE TOTAL PLANTACIONES PYMP POR REGIÓN 2004 Y 2010

\section{Plantaciones de Eucaliptos según Tipo de Propietario}

El segmento PYMP ha sido gravitante en el incremento de las plantaciones de eucaliptos y al 2011 poseía el $51 \%$ de la superficie de ellas, especialmente de aquellas de Eucalyptus globulus en que este segmento de propietarios controlaba el $60 \%$ de la superficie plantada (Cuadro $\mathrm{N}^{\circ}$ 2). En el caso de las plantaciones de Eucalyptus nitens, el segmento PYMP tiene el $29 \%$ de la superficie plantada.

Cuadro $\mathbf{N}^{\circ} 2$

SUPERFICIE PLANTACIONES DE EUCALIPTO SEGÚN TIPO PROPIETARIO, ESPECIE Y CLASE DE EDAD (2011)

\begin{tabular}{|c|c|c|c|c|c|}
\hline \multirow{2}{*}{ Edad } & \multirow{2}{*}{ Empresas } & \multicolumn{3}{|c|}{ PYMP } & \multirow{2}{*}{ Total } \\
\hline & & Medianos & Pequeños & Total & \\
\hline (Años) & \multicolumn{5}{|c|}{ (ha) } \\
\hline \multicolumn{6}{|c|}{ Eucalyptus globulus } \\
\hline 0 a 4 & 65.698 & 18.843 & 57.346 & 76.189 & 141.887 \\
\hline 5 a 9 & 79.590 & 28.382 & 123.322 & 151.704 & 231.294 \\
\hline 10 a 14 & 48.776 & 12.633 & 45.611 & 58.244 & 107.020 \\
\hline 15 a 19 & 8.379 & 4.133 & 16.926 & 21.059 & 29.438 \\
\hline 20 a 24 & 4.011 & 1.561 & 5.218 & 6.779 & 10.790 \\
\hline$>24$ & 1.024 & 233 & 1.845 & 2.078 & 3.102 \\
\hline Total & 207.478 & 65.785 & 250.268 & 316.053 & 523.531 \\
\hline \multicolumn{6}{|c|}{ Eucalyptus nitens } \\
\hline 0 a 4 & 64.149 & 6.128 & 13.617 & 19.745 & 83.894 \\
\hline 5 a 9 & 25.482 & 8.453 & 16.282 & 24.735 & 50.217 \\
\hline 10 a 14 & 37.725 & 2.045 & 9.222 & 11.267 & 48.992 \\
\hline 15 a 19 & 25.547 & 2.374 & 3.699 & 6.073 & 31.620 \\
\hline 20 a 24 & 1.685 & 136 & 137 & 273 & 1.958 \\
\hline$>24$ & 118 & 20 & 10 & 30 & 148 \\
\hline Total & 154.706 & 19.156 & 42.967 & 62.123 & 216.829 \\
\hline TOTAL & 362.184 & 84.941 & 293.235 & 378.176 & 740.360 \\
\hline
\end{tabular}

(Fuente: INFOR, 2013) 
Destaca que solo los pequeños propietarios poseen el $48 \%$ de las plantaciones de Eucalyptus globulus, el $20 \%$ de aquellas de Eucalyptus nitens y casi el $40 \%$ del total plantado con eucaliptos en el país. Algo más del $50 \%$ de las plantaciones del segmento PYMP corresponde a plantaciones de eucaliptos.

El comportamiento de pequeños y medianos propietarios respecto de las especies que emplean en sus plantaciones ha variado en las últimas décadas. En los años 80 predominó en sus plantaciones pino radiata, posteriormente se incrementó en ellas la participación de eucaliptos y solo entre 2002 y 2011 este segmento plantó más de 270 mil hectáreas con eucaliptos, lo que representa más del $70 \%$ de su patrimonio actual de plantaciones de eucaliptos a 2011 (Cuadro $\mathrm{N}^{\circ}$ 2 y Figura $N^{\circ} 4$ ) y el $36 \%$ de las plantaciones totales de eucaliptos al mismo año.

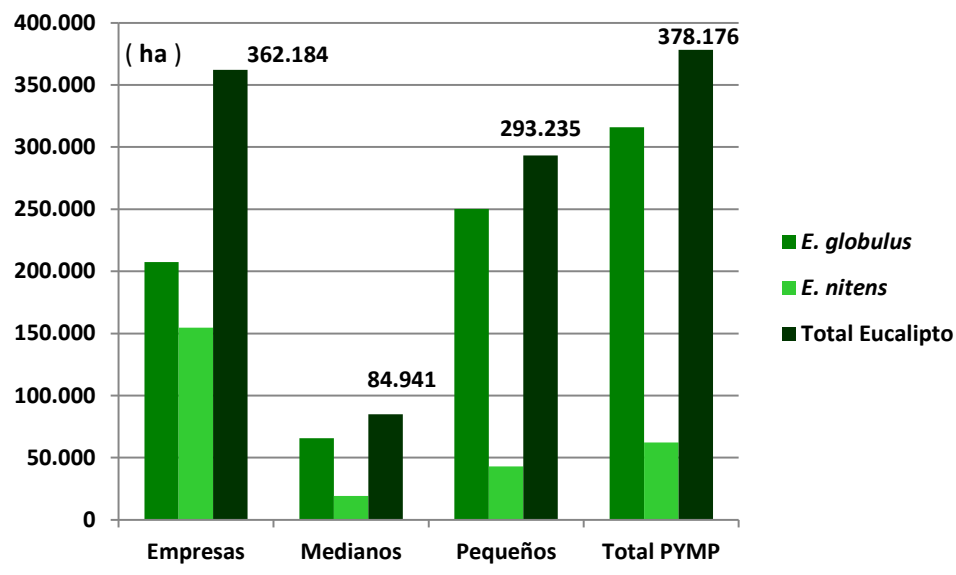

Figura $N^{\circ} 4$

SUPERFICIE PLANTACIONES DE EUCALIPTO SEGÚN TIPO PROPIETARIO Y ESPECIE (2011)

\section{MATERIAL Y MÉTODO}

El análisis de cambio de especies en las plantaciones del segmento PYMP se realizó en base a dos fuentes de información; las actualizaciones anuales de plantaciones de INFOR y las intenciones de reforestación presentadas a la Corporación Nacional Forestal (CONAF), en cumplimiento de la legislación forestal vigente en lo relativo a la obligatoriedad de reforestación después de cortas de cosecha, para las regiones de O'Higgins a Los Lagos.

Respecto de la información de INFOR, la variable a emplear es la razón entre la superficie acumulada de eucalipto (Eucalyptus globulus, E. nitens y E. spp) y la superficie acumulada de pino radiata (PEu / PPr), para cada año del período 1991 - 2010. Este análisis considera forestación y reforestación y la relación PEu / PPr se establece sobre las superficies totales existentes anualmente.

$$
\operatorname{PEu} / \operatorname{PPr}=\sum \mathrm{S}_{\text {Eu Región Año }} / \mathrm{S}_{\mathrm{Pr} \text { Región Año }}
$$

En cuanto a la segunda fuente de información, se efectúa un análisis similar respecto de la reforestación que registra el segmento PYMP, instancia en la que se produce el cambio de especies. La variable a emplear en este caso para dimensionar el cambio de especies es la razón 
superficie reforestada con eucalipto y la superficie reforestada con pino radiata (REu / RPr) en el período 2006 a 2011.

$$
\operatorname{REu} / \operatorname{RPr}=\sum S_{\text {Eu Región Año }} / S_{\text {Pr Región Año }}
$$

\section{ANÁLISIS DE LA INFORMACIÓN Y RESULTADOS}

\section{Relación PEu / PPr Plantaciones PYMP}

En todas las regiones consideradas en el estudio se aprecia un aumento de la superficie plantada con eucalipto respecto de la plantada con pino radiata y con el transcurrir de los años del periodo analizado las magnitudes de este aumento se hacen más importantes, principalmente desde la región del Bío Bío al sur (Cuadro N³).

\section{Cuadro $\mathrm{N}^{\circ} 3$}

PLANTACIONES ACUMULADAS PYMP POR AÑO Y REGIÓN RAZÓN PEu / PPr

\begin{tabular}{|r|r|r|r|r|r|r|}
\hline \multirow{2}{*}{ Año } & \multicolumn{6}{|c|}{ Región } \\
\cline { 2 - 7 } & O’Higgins & Maule & Bío Bío & Araucanía & \multicolumn{1}{c|}{$\begin{array}{c}\text { Los } \\
\text { Ríos }\end{array}$} & $\begin{array}{c}\text { Los } \\
\text { Lagos }\end{array}$ \\
\hline 1991 & & & & 0,10 & 0,21 & 0,15 \\
1995 & 0,29 & & & & \\
1996 & & 0,08 & & & & \\
1998 & & & 0,21 & & \\
2001 & & & 0,00 & 0,41 & & \\
2002 & & 0,10 & 0,37 & 0,00 & 1,09 & 2,76 \\
2004 & 0,32 & 0,11 & 0,41 & 0,76 & 2,44 & 3,17 \\
2005 & 0,46 & 0,17 & 0,66 & 1,06 & 2,86 & 2,93 \\
2006 & 0,56 & 0,18 & 0,72 & 1,36 & 3,17 & 2,87 \\
2007 & 0,59 & 0,19 & 0,78 & 1,55 & 3,62 & 3,04 \\
2008 & 0,61 & 0,18 & 0,81 & 1,61 & 3,89 & 3,52 \\
2009 & 0,63 & 0,19 & 0,83 & 1,71 & 3,67 & 3,85 \\
2010 & 0,68 & 0,19 & 0,89 & 1,77 & 3,82 & 3,94 \\
\hline
\end{tabular}

En la región del Maule se aprecia una cierta estabilidad en la relación de superficies y es la región con la razón PEu / PPr más baja, en tanto que las regiones de Los Ríos y Los Lagos muestran las tasas de incremento más altas.

La superficies acumuladas de plantaciones por región, año y especie, que dan origen a las razones PEu / PPr en cada caso, se pueden apreciar en Apéndice $\mathrm{N}^{\circ} 1$.

En la Figura $N^{\circ} 5$ se muestra gráficamente las variaciones anuales de la razón $\mathrm{PEu} / \mathrm{PPr}$ para cada una de las regiones y se aprecia que el promedio calculado (línea de color rojo) indica una tendencia creciente para esta variable, en especial a partir del año 2001. 


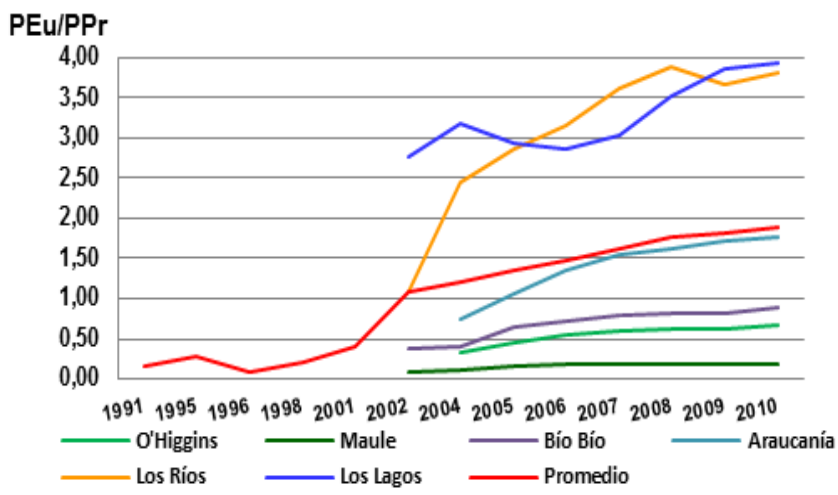

Figura $N^{\circ} 5$

RAZÓN PEu / PPr EN PLANTACIONES PYMP POR AÑOS Y REGIONES

\section{Relación REu/RPr Reforestaciones PYMP}

La superficie total reforestada por el segmento PYMP para el período 2006-2011 en las regiones consideradas es de 163.251 ha, el $56,6 \%$ de esta superficie corresponde a pino radiata, el $41,2 \%$ a eucalipto y el $2,2 \%$ a otras especies (Cuadro $N^{\circ} 4$ y Figura $N^{\circ} 6$ ).

La reforestación del segmento se mantiene constante, aunque muestra una leve tendencia decreciente, el empleo de pino aparece con una tendencia claramente decreciente, a la inversa el uso de eucaliptos en la reforestación aparece en aumento.

\section{Cuadro $\mathrm{N}^{\circ} 4$ \\ SUPERFICIE REFORESTADA PYMP POR ESPECIE Y AÑO}

\begin{tabular}{|c|c|c|c|c|c|c|c|c|}
\hline \multirow{2}{*}{ Año } & \multicolumn{2}{|c|}{ Pino } & \multicolumn{2}{c|}{ Eucaliptos } & \multicolumn{2}{c|}{ Otras } & \multicolumn{2}{c|}{ Total } \\
\cline { 2 - 8 } & (ha) & (\%) & (ha) & (\%) & (ha) & (\%) & (ha) & (\%) \\
\hline
\end{tabular}

\begin{tabular}{|l|r|r|r|r|r|r|r|r|}
\hline 2006 & 18.407 & 62,5 & 10.218 & 34,7 & 832 & 2,8 & $\mathbf{2 9 . 4 5 8}$ & 100 \\
\hline 2007 & 14.718 & 59,2 & 9.763 & 39,3 & 383 & 1,5 & $\mathbf{2 4 . 8 6 4}$ & 100 \\
\hline 2008 & 16.484 & 60,6 & 9.936 & 36,5 & 771 & 2,8 & $\mathbf{2 7 . 1 9 1}$ & 100 \\
\hline 2009 & 16.007 & 52,5 & 14.108 & 46,2 & 403 & 1,3 & $\mathbf{3 0 . 5 1 8}$ & 100 \\
\hline 2010 & 12.989 & 52,6 & 11.178 & 45,3 & 525 & 2,1 & $\mathbf{2 4 . 6 9 1}$ & 100 \\
\hline 2011 & 13.751 & 51,8 & 12.066 & 45,5 & 711 & 2,7 & $\mathbf{2 6 . 5 2 8}$ & 100 \\
\hline
\end{tabular}

\begin{tabular}{|c|c|c|c|c|c|c|c|c|}
\hline Total & 92.357 & 56,6 & 67.269 & 41,2 & 3.626 & 2,2 & 163.251 & 100 \\
\hline Promedio & 15.393 & & 11.212 & & 604 & & 27.209 & \\
\hline
\end{tabular}




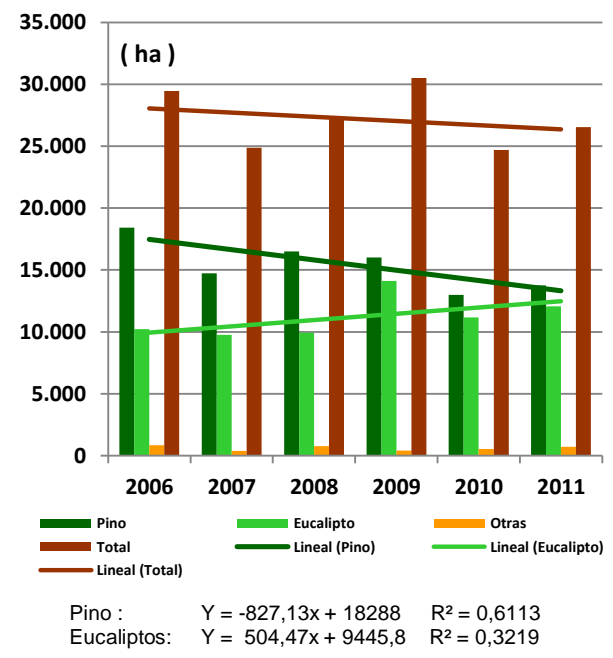

\section{Figura $\mathrm{N}^{\circ} 6$ \\ REFORESTACIONES PYMP POR ESPECIE Y AÑO PERÍODO 2006-2011}

Este segmento de propietarios ha estado reforestando en el período indicado a una tasa media anual de algo más de 27 mil hectáreas, dentro de la cual la participación de pino se ha reducido de 63 a $57 \%$ en tanto que la de eucaliptos se ha incrementado de 35 a $46 \%$.

Lo anterior indica que, con una tendencia creciente, estos propietarios al reponer superficies cosechadas de pino están cambiando de especie y reforestando con eucaliptos.

Las regresiones realizadas para las curvas de tendencias señalan que la tasa de disminución de reforestación con pino sería de 827 ha por año, en tanto que la de aumento de eucaliptos se situaría en 504 ha anuales (Figura $N^{\circ}$ 6).

Destaca el año 2009, en el cual la reforestación con eucalipto alcanzó su nivel más alto, muy cercano a la reforestación con pino, manteniéndose después una diferencia semejante entre una y otra.

Las superficies de reforestaciones del segmento, por región, especie y año, pueden ser apreciadas en Apéndice $\mathrm{N}^{\circ} 2$.

En cuanto a las regiones, las que presentan las mayores tendencias al cambio de especies en el período son O’Higgins, Bío Bío, Araucanía y Los Ríos, en especial la tercera de ellas (Figura $\mathrm{N}^{\circ} 7$ ). 

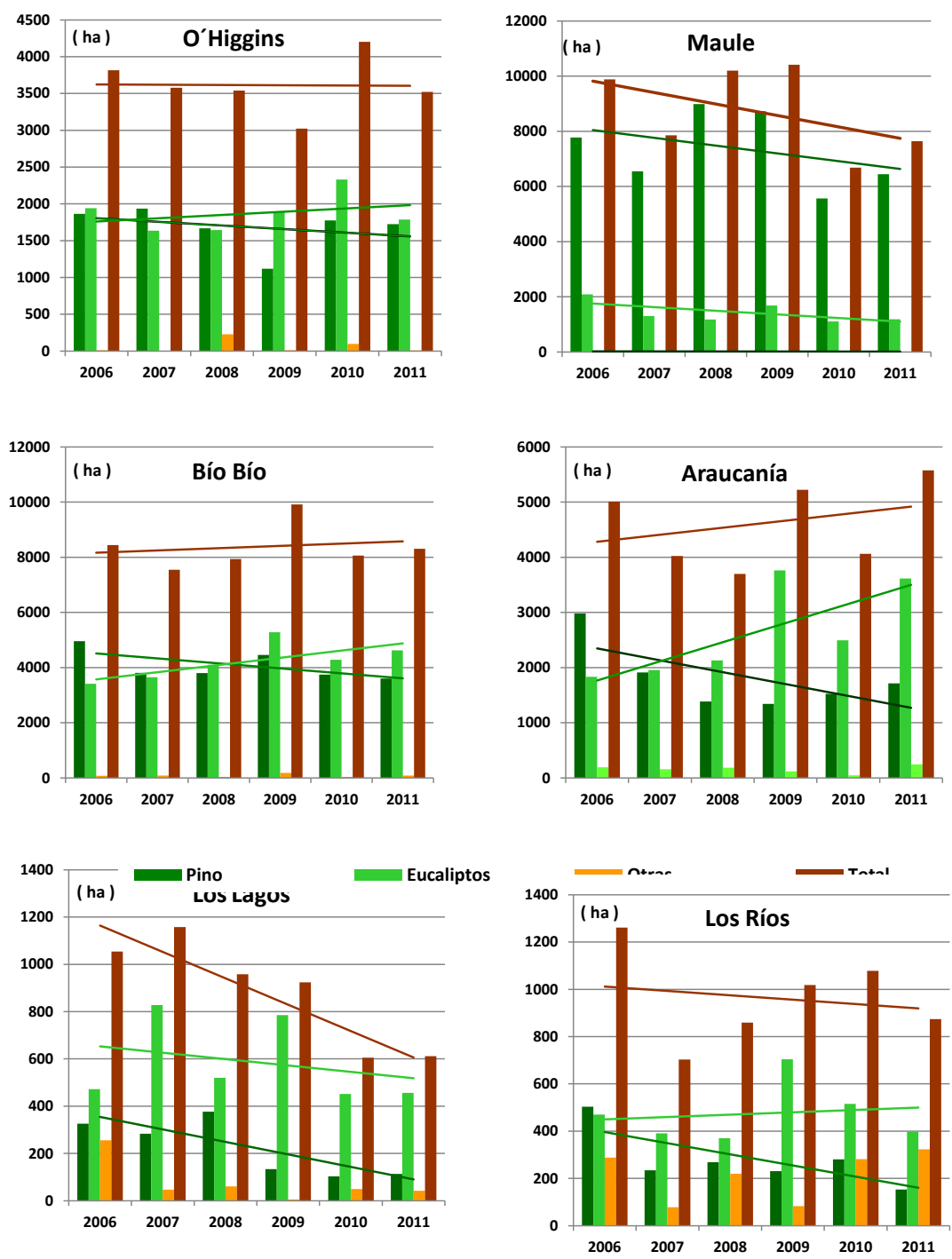

Figura $\mathrm{N}^{\circ} 7$

REFORESTACIONES PYMP POR REGIÓN, ESPECIE Y AÑO PERÍODO 2006-2011

En la región del Maule se aprecian tendencias decrecientes en la reforestación, tanto para pino como para eucaliptos, pero en el caso de eucaliptos es mucho más leve. También es decreciente la tendencia para ambas especies en la región de los Lagos, aun cuando las tasas de reforestación con eucaliptos en los años 2007 y 2009 fueron importantes. 
Para dimensionar las tendencias de cambio de especies en las plantaciones forestales se usó primero la razón PEu / PPr respecto de la superficie acumulada de plantaciones año a año del segmento de propietarios PYMP, la cual evoluciona de acuerdo las superficies cosechadas, forestadas y reforestadas anualmente y las especie involucradas en cada caso.

Ahora se utiliza información sobre la especie empleada en reforestación, que es la reposición de superficie cosechada, por tanto la instancia donde se produce el cambio de especie. La razón REu / RPr relaciona las superficies reforestadas con pino y eucaliptos e indica la superficie reforestada con eucaliptos por cada unidad de superficie (ha) reforestada con pino. Esta relación se analiza a continuación.

La razón REu / RPr para el total de las regiones consideradas tiene un leve aumento desde el año 2006 y permanece relativamente constante y próxima a 1 desde el año 2009 (Cuadro $\mathrm{N}^{\circ} 5$ y Figura $\mathrm{N}^{\circ}$ 8). Esto indica que en promedio para las 6 regiones en el periodo 2006-2009 se incrementa la reforestación con eucaliptos en desmedro de aquella con pino, pero aún es mayor la reforestación con pino.

\section{Cuadro $\mathrm{N}^{\circ} 5$}

RAZÓN REu / RPr EN REFORESTACIONES PYMP POR REGIONES Y AÑOS

\begin{tabular}{|c|c|c|c|c|c|c|}
\hline \multirow{2}{*}{ Región } & \multicolumn{6}{|c|}{ REu / RPr por Región y Año } \\
\hline & 2006 & 2007 & 2008 & 2009 & 2010 & 2011 \\
\hline O’Higgins & 1,04 & 0,84 & 0,99 & 1,69 & 1,31 & 1,04 \\
\hline Maule & 0,27 & 0,20 & 0,13 & 0,19 & 0,20 & 0,18 \\
\hline Bío Bío & 0,69 & 0,96 & 1,08 & 1,19 & 1,14 & 1,29 \\
\hline La Araucanía & 0,61 & 1,02 & 1,54 & 2,81 & 1,64 & 2,11 \\
\hline Los Ríos & 0,93 & 1,67 & 1,37 & 3,05 & 1,83 & 2,61 \\
\hline Los Lagos & 1,45 & 2,93 & 1,38 & 5,85 & 4,39 & 4,04 \\
\hline Total & 0,56 & 0,66 & 0,60 & 0,88 & 0,86 & 0,88 \\
\hline
\end{tabular}

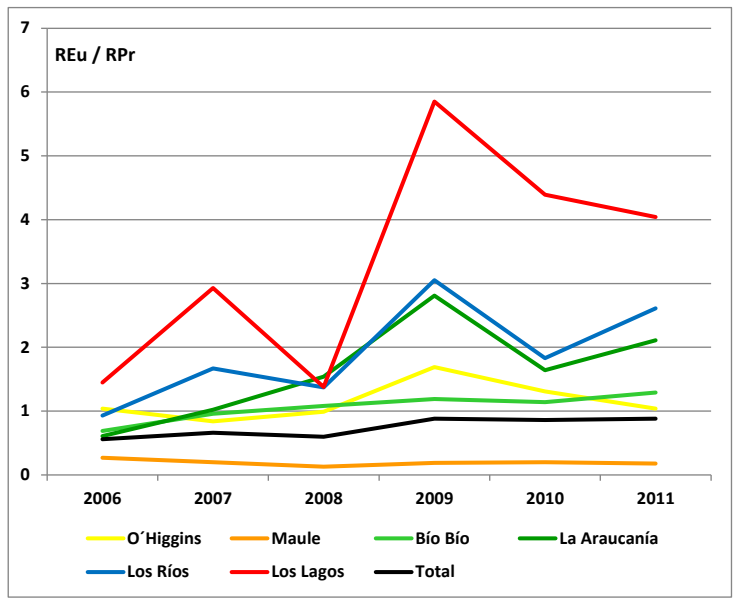

Figura $\mathrm{N}^{\circ} 8$

RAZÓN REu / RPr EN REFORESTACIONES PYMP REGIONES Y AÑOS 
Se observa que las regiones con mayor alza de la razón REu / RPr corresponden a Los Lagos, Los Ríos y La Araucanía. En el año 2006 solo en la Región de Los Lagos la reforestación con eucalipto superaba claramente a aquella con pino, pero después la tendencia al cambio de especies se hace creciente; en 2009 en Los Lagos la reforestación con eucaliptos sextuplica a aquella con pino, en tanto que en Los Ríos y en La Araucanía la triplica. Para el año 2011 las diferencias disminuyen, pero sigue siendo considerablemente mayor la participación de eucaliptos en la reforestación en estas regiones.

En la región del Bío Bío la reforestación con una y otra especie es similar en 2006, pero en 2007 empieza a crecer la participación de eucaliptos y desde 2008 supera a la de pino y se mantiene esta tendencia hasta 2011.

En la región de O’Higgins la tendencia indica valores similares para una y otra especie de 2006 a 2008, pero en 2009 eucalipto supera claramente a pino, situación que se mantiene en 2010 y para 2011 tienden a igualarse las cifras nuevamente.

La región del Maule en tanto muestra una situación más o menos constante durante el período y la reforestación con pino continúa superando claramente a aquella con eucaliptos.

\section{CONCLUSIONES}

En todas las regiones consideradas en el estudio se aprecia un aumento de la superficie plantada con eucalipto respecto de la plantada con pino radiata y con el transcurrir de los años del periodo analizado las magnitudes de este incremento se hacen más importantes, principalmente desde la región del Bío Bío al sur.

Las plantaciones de eucalipto aumentaron en relación a las de pino, fuertemente en las regiones del sur, por efecto del incremento de la participación de eucaliptos, tanto en forestaciones como por el reemplazo de pino en reforestaciones.

La excepción se produce en la región del Maule, en donde la participación de eucaliptos se mantiene relativamente constante y predomina claramente pino.

En términos de superficie, los mayores cambios desde plantaciones de pino hacia eucalipto en PYMP se produjeron en las regiones del Bío Bío y Araucanía.

En las plantaciones de PYMP existe un aumento en la tendencia a reforestar con eucalipto y una disminución a hacerlo con pino. Siendo aparentemente más rápida la disminución de pino que el aumento de eucalipto. Esto último debido, en parte, al cambio de especie y al cumplimiento del plan de manejo, ya que de acuerdo al DL $N^{\circ} 701$ quien cosecha tiene un plazo de tres años para reforestar.

Las regiones con los indicadores más altos a reforestar con eucalipto son Los Lagos, Los Ríos y Araucanía, en menor proporción Bío Bío y O’Higgins. La región del Maule en tanto es la que presenta los indicadores más bajos de participación de reforestación con eucalipto.

Es interesante destacar que en ambos análisis; razón de superficie acumulada de eucalipto y pino, incluida la forestación y la reforestación, basado en información INFOR, y reforestación eucalipto y pino, basada en información CONAF, se encuentra un aumento relativo de las plantaciones de especies del género Eucalyptus en las plantaciones de PMYP.

En este aumento de la participación de eucalipto destacan las regiones de Araucanía, Los Ríos y Los Lagos. En términos absolutos de cambios de especie, las mayores superficies se concentraron en las regiones de Bío Bío y Araucanía. 
Diversos factores están contribuyendo a estas tendencias de cambio de especies, todos ellos de especial interés para el segmento PYMP. Entre los principales están el rápido crecimiento de los eucaliptos y su consecuente menor rotación, el vigoroso rebrote de tocones de Eucalyptus globulus para constituir un monte bajo y evitar así la necesidad de una nueva plantación, el mercado de madera para pulpa y su precio, la adaptabilidad a sectores de heladas particularmente de Eucalyptus nitens, los bajos costos de manejo de las plantaciones de eucaliptos que hoy se destinan a pulpa en corta rotación y sin intervenciones de raleos ni podas, y los incentivos estatales para la forestación, aún vigentes para el período analizado.

A lo anterior se suma sin duda la abundante información que INFOR ha difundido de sus líneas de investigación sobre eucaliptos, desde la introducción al país de una cantidad de especies del género Eucalyptus y la evaluación de su adaptación y crecimiento en las diferentes regiones hasta diversas investigaciones posteriores respecto de la silvicultura y manejo de las principales de estas, en lo que se refiere a manejo y tratamiento de semillas, viverización de plantas en viveros, técnicas de establecimiento de plantaciones, espaciamiento de estas, propiedades físicas y mecánicas de la madera, utilización y otras, sin contar los intensos programas de mejoramiento genético implementados posteriormente.

Cabe destacar por ejemplo que, producto de investigaciones de INFOR, fueron masificadas en el país las técnicas de producción de plantas en contenedores en ambientes controlados, que condujeron a una segura y eficiente producción de material de plantación de calidad, y las técnicas intensivas de establecimiento de plantaciones, que combinaron una fuerte preparación de suelos, control de competencia de malezas y fertilización inicial, contribuyendo a mejoras sustanciales en el prendimiento, desarrollo inicial y supervivencia en la forestación y reforestación con estas especies.

\section{REFERENCIAS}

INFOR, 2005. Disponibilidad de Madera de Plantaciones de Pino Radiata en Chile 2003-2032. 103 pp. Instituto Forestal, Informe Técnico $N^{\circ} 170$. Santiago, Chile.

INFOR, 2007. Disponibilidad de Madera de Eucalyptus en Chile 2006-2025. 63 pp. Instituto Forestal, Informe Técnico $\mathrm{N}^{\circ}$ 173. Valdivia, Chile.

INFOR, 2011. Los Recursos Forestales en Chile, Informe Final. Inventario Continuo de Bosques Nativos y Actualización de Plantaciones Forestales. 298 p.

INFOR, 2013. Disponibilidad de Madera de Plantaciones de Pino Radiata y Eucalipto (2010-2040). 115 p. Instituto Forestal, Informe Técnico Nº 194

INFOR, 2014. Anuario Forestal 2014. Boletín Estadístico № 144. Instituto Forestal. 159 p. 
APÉNDICE N ${ }^{\circ}$

SUPERFICIE ACUMULADA PLANTACIONES POR REGIÓN Y ESPECIE PYMP 1991-2010

Región de Ohiggins

\begin{tabular}{|c|c|c|c|c|}
\hline \multirow{2}{*}{ Año } & Eucalyptus spp. & Pinus radiata & Total & \multirow{2}{*}{$\begin{array}{c}\mathrm{PEu} / \mathrm{Pr} \\
(-)\end{array}$} \\
\hline & \multicolumn{3}{|c|}{ ( ha ) } & \\
\hline 2010 & 39.355 & 57.787 & 97.142 & 0,68 \\
\hline 2009 & 35.828 & 57.277 & 93.105 & 0,63 \\
\hline 2008 & 34.820 & 56.701 & 91.521 & 0,61 \\
\hline 2007 & 34.115 & 58.017 & 92.132 & 0,59 \\
\hline 2006 & 31.840 & 56.897 & 88.737 & 0,56 \\
\hline 2005 & 25.527 & 55.914 & 81.441 & 0,46 \\
\hline 2004 & 17.117 & 53.157 & 70.274 & 0,32 \\
\hline 1995 & 16.930 & 57.931 & 74.861 & 0,29 \\
\hline
\end{tabular}

Región del Maule

\begin{tabular}{|r|r|r|r|r|}
\hline \multirow{2}{*}{ Año } & Eucalyptus spp. & Pinus radiata & \multicolumn{1}{c|}{ Total } & \multicolumn{2}{c|}{$\begin{array}{c}\text { PEu/Pr } \\
\text { ( - ) }\end{array}$} \\
\hline & \multicolumn{3}{|c|}{ ha $)$} & 0,19 \\
\hline 2010 & 31.833 & 167.147 & 198.980 & 0,19 \\
\hline 2009 & 31.407 & 165.787 & 197.194 & 0,18 \\
\hline 2008 & 29.349 & 159.288 & 188.637 & 0,19 \\
\hline 2007 & 29.737 & 155.443 & 185.180 & 0,18 \\
\hline 2006 & 27.299 & 148.157 & 175.456 & 0,17 \\
\hline 2005 & 22.670 & 137.370 & 160.040 & 0,11 \\
\hline 2004 & 13.689 & 122.949 & 136.638 & 0,10 \\
\hline 2002 & 11.159 & 113.556 & 124.715 & 0,08 \\
\hline 1996 & 15.306 & 189.299 & 204.605 & \\
\hline
\end{tabular}

Región del Bío Bío

\begin{tabular}{|c|r|r|r|r|}
\hline \multirow{2}{*}{ Año } & Eucalyptus spp. & Pinus radiata & \multicolumn{1}{c|}{ Total } & \multirow{2}{*}{$\begin{array}{c}\text { PEu/Pr } \\
\text { ( - ) }\end{array}$} \\
\cline { 2 - 4 } & \multicolumn{2}{|c|}{ ( ha ) } & 0,89 \\
\hline 2010 & 107.013 & 120.081 & 227.094 & 0,83 \\
\hline 2009 & 100.384 & 121.282 & 221.666 & 0,81 \\
\hline 2008 & 93.905 & 115.711 & 209.616 & 0,78 \\
\hline 2007 & 91.750 & 117.160 & 208.910 & 0,72 \\
\hline 2006 & 84.261 & 116.924 & 201.185 & 0,66 \\
\hline 2005 & 69.690 & 105.734 & 175.424 & 0,41 \\
\hline 2004 & 44.694 & 109.679 & 154.373 & 0,37 \\
\hline 2002 & 43.071 & 116.414 & 159.485 & 0,21 \\
\hline 1998 & 36.864 & 174.448 & 211.312 & \\
\hline
\end{tabular}


Región de La Araucanía

\begin{tabular}{|r|r|r|r|r|}
\hline \multirow{2}{*}{ Año } & Eucalyptus spp. & Pinus radiata & \multicolumn{1}{c|}{ Total } & \multirow{2}{*}{$\begin{array}{c}\text { PEu/Pr } \\
\text { ( - ) }\end{array}$} \\
\hline & \multicolumn{3}{|c|}{ ( ha ) } & 1,77 \\
\hline 2010 & 105.177 & 59.553 & 164.730 & 1,71 \\
\hline 2009 & 100.284 & 58.710 & 158.994 & 1,61 \\
\hline 2008 & 84.550 & 52.508 & 137.058 & 1,55 \\
\hline 2007 & 86.480 & 55.666 & 142.146 & 1,36 \\
\hline 2006 & 79.018 & 58.079 & 137.097 & 1,06 \\
\hline 2005 & 61.440 & 57.757 & 119.197 & 0,76 \\
\hline 2004 & 42.003 & 55.570 & 97.573 & 0,41 \\
\hline 2001 & 34.423 & 83.677 & 118.100 & 0,10 \\
\hline 1991 & 8.869 & 91.147 & 100.016 & \\
\hline
\end{tabular}

Región de Los Ríos

\begin{tabular}{|r|r|r|r|r|}
\hline \multirow{2}{*}{ Año } & Eucalyptus spp. & Pinus radiata & \multicolumn{1}{c|}{ Total } & \multirow{2}{*}{$\begin{array}{c}\text { PEu/Pr } \\
\text { ( - ) }\end{array}$} \\
\cline { 2 - 4 } & \multicolumn{3}{|c|}{ ( ha ) } & 3,82 \\
\hline 2010 & 28.191 & 7.376 & 35.567 & 3,67 \\
\hline 2009 & 25.402 & 6.930 & 32.332 & 3,89 \\
\hline 2008 & 26.064 & 6.705 & 32.769 & 3,62 \\
\hline 2007 & 25.657 & 7.088 & 32.745 & 3,17 \\
\hline 2006 & 24.283 & 7.664 & 31.947 & 2,86 \\
\hline 2005 & 21.024 & 7.345 & 28.369 & 2,47 \\
\hline 2004 & 20.280 & 8.203 & 28.483 & 1,09 \\
\hline 2002 & 16.562 & 15.242 & 31.804 & 0,21 \\
\hline 1991 & 3.111 & 14.584 & 17.695 & \\
\hline
\end{tabular}

Región de Los Lagos

\begin{tabular}{|c|c|c|c|c|}
\hline \multirow{2}{*}{ Año } & Eucalyptus spp. & Pinus radiata & Total & \multirow{2}{*}{$\begin{array}{c}\text { PEu/Pr } \\
(-)\end{array}$} \\
\hline & \multicolumn{3}{|c|}{ (ha) } & \\
\hline 2010 & 18.400 & 4.670 & 23.070 & 3,94 \\
\hline 2009 & 18.383 & 4.771 & 23.154 & 3,85 \\
\hline 2008 & 16.802 & 4.779 & 21.581 & 3,52 \\
\hline 2007 & 17.529 & 5.758 & 23.287 & 3,04 \\
\hline 2006 & 14.383 & 5.010 & 19.393 & 2,87 \\
\hline 2005 & 15.064 & 5.137 & 20.201 & 2,93 \\
\hline 2004 & 15.431 & 4.871 & 20.302 & 3,17 \\
\hline 2002 & 16.456 & 5.970 & 22.426 & 2,76 \\
\hline 1991 & 1.172 & 7.894 & 9.066 & 0,15 \\
\hline
\end{tabular}




\section{APÉNDICE $\mathrm{N}^{\circ} 2$ \\ SUPERFICIE REFORESTADA POR REGIÓN Y ESPECIE PYMP \\ 2006-2011}

Región de O’Higgins

\begin{tabular}{|c|r|r|r|r|r|r|r|r|}
\hline \multirow{2}{*}{ Año } & \multicolumn{2}{|c|}{ Pinus radiata } & \multicolumn{2}{c|}{ Eucalyptus spp } & \multicolumn{2}{c|}{ Otras } & \multicolumn{2}{|c|}{ Total } \\
\cline { 2 - 9 } & ( ha $)$ & \multicolumn{1}{c|}{ ( \%) } & \multicolumn{1}{c|}{ ( ha $)$} & \multicolumn{1}{c|}{ ( \%) } & ( ha ) & ( \%) & ( ha ) & ( \%) \\
\hline 2006 & 1.863 & 48,8 & 1.943 & 50,9 & 10 & 0,3 & $\mathbf{3 . 8 1 5}$ & 100 \\
\hline 2007 & 1.936 & 54,1 & 1.636 & 45,7 & 6 & 0,2 & $\mathbf{3 . 5 7 8}$ & 100 \\
\hline 2008 & 1.668 & 47,1 & 1.644 & 46,4 & 228 & 6,4 & $\mathbf{3 . 5 4 1}$ & 100 \\
\hline 2009 & 1.120 & 37,1 & 1.890 & 62,5 & 12 & 0,4 & $\mathbf{3 . 0 2 3}$ & 100 \\
\hline 2010 & 1.774 & 42,2 & 2.331 & 55,5 & 96 & 2,3 & $\mathbf{4 . 2 0 1}$ & 100 \\
\hline 2011 & 1.724 & 49,0 & 1.788 & 50,8 & 9 & 0,3 & $\mathbf{3 . 5 2 2}$ & 100 \\
\hline Total & 10.085 & 46,5 & 11.232 & 51,8 & 361 & 1,7 & $\mathbf{2 1 . 6 7 9}$ & 100 \\
\hline Promedio & 1.681 & & 1.872 & & 60 & & $\mathbf{3 . 6 1 3}$ & \\
\hline
\end{tabular}

\section{Región del Maule}

\begin{tabular}{|c|c|c|c|c|c|c|c|c|}
\hline \multirow{2}{*}{ Año } & \multicolumn{2}{|c|}{ Pinus radiata } & \multicolumn{2}{|c|}{ Eucalyptus spp } & \multicolumn{2}{|c|}{ Otras } & \multicolumn{2}{|c|}{ Total } \\
\hline & (ha) & $(\%)$ & (ha ) & $(\%)$ & ( ha ) & $(\%)$ & ( ha ) & $(\%)$ \\
\hline 2006 & 7.779 & 78,7 & 2.089 & 21,1 & 15 & 0,1 & 9.883 & 100 \\
\hline 2007 & 6.546 & 83,3 & 1.302 & 16,6 & 10 & 0,1 & 7.858 & 100 \\
\hline 2008 & 8.985 & 88,1 & 1.174 & 11,5 & 42 & 0,4 & 10.201 & 100 \\
\hline 2009 & 8.725 & 83,8 & 1.685 & 16,2 & 4 & 0,0 & 10.414 & 100 \\
\hline 2010 & 5.563 & 83,2 & 1.109 & 16,6 & 12 & 0,2 & 6.684 & 100 \\
\hline 2011 & 6.448 & 84,3 & 1.183 & 15,5 & 13 & 0,2 & 7.644 & 100 \\
\hline Total & 44.046 & 83,6 & 8.542 & 16,2 & 95 & 0,2 & 52.683 & 100 \\
\hline Promedio & 7.341 & & 1.424 & & 16 & & 8.781 & \\
\hline
\end{tabular}

\section{Región del Bio Bio}

\begin{tabular}{|c|c|c|c|c|c|c|c|c|}
\hline \multirow{2}{*}{ Año } & \multicolumn{2}{|c|}{ Pinus radiata } & \multicolumn{2}{|c|}{ Eucalyptus spp } & \multicolumn{2}{|c|}{ Otras } & \multicolumn{2}{|c|}{ Total } \\
\hline & (ha) & $(\%)$ & ( ha ) & $(\%)$ & ( ha ) & $(\%)$ & (ha ) & $(\%)$ \\
\hline 2006 & 4.955 & 58,7 & 3.411 & 40,4 & 73 & 0,9 & 8.438 & 100 \\
\hline 2007 & 3.807 & 50,5 & 3.650 & 48,4 & 86 & 1,1 & 7.543 & 100 \\
\hline 2008 & 3.798 & 47,9 & 4.099 & 51,7 & 36 & 0,5 & 7.933 & 100 \\
\hline 2009 & 4.456 & 44,9 & 5.281 & 53,2 & 181 & 1,8 & 9.919 & 100 \\
\hline 2010 & 3.744 & 46,4 & 4.279 & 53,1 & 39 & 0,5 & 8.063 & 100 \\
\hline 2011 & 3.598 & 43,3 & 4.626 & 55,7 & 81 & 1,0 & 8.304 & 100 \\
\hline Total & 24.358 & 48,5 & 25.347 & 50,5 & 495 & 1,0 & 50.200 & 100 \\
\hline Promedio & 4.060 & & 4.224 & & 83 & & 8.367 & \\
\hline
\end{tabular}


Región de la Araucanía

\begin{tabular}{|c|c|c|c|c|c|c|c|c|}
\hline \multirow{2}{*}{ Año } & \multicolumn{2}{|c|}{ Pinus radiata } & \multicolumn{2}{|c|}{ Eucalyptus spp } & \multicolumn{2}{|c|}{ Otras } & \multicolumn{2}{|c|}{ Total } \\
\hline & ( ha ) & $(\%)$ & ( ha ) & $(\%)$ & ( ha ) & $(\%)$ & ( ha ) & $(\%)$ \\
\hline 2006 & 2.982 & 59,6 & 1.834 & 36,6 & 191 & 3,8 & 5.007 & 100 \\
\hline 2007 & 1.912 & 47,5 & 1.956 & 48,6 & 156 & 3,9 & 4.023 & 100 \\
\hline 2008 & 1.386 & 37,5 & 2.128 & 57,5 & 184 & 5,0 & 3.699 & 100 \\
\hline 2009 & 1.341 & 25,7 & 3.762 & 72,1 & 118 & 2,3 & 5.221 & 100 \\
\hline 2010 & 1.524 & 37,5 & 2.492 & 61,3 & 46 & 1,1 & 4.062 & 100 \\
\hline 2011 & 1.715 & 30,8 & 3.616 & 64,9 & 243 & 4,4 & 5.574 & 100 \\
\hline Total & 10.859 & 39,4 & 15.787 & 57,2 & 939 & 3,4 & 27.586 & 100 \\
\hline Promedio & 1.810 & & 2.631 & & 156 & & 4.598 & \\
\hline
\end{tabular}

\section{Región de Los Ríos}

\begin{tabular}{|c|c|c|c|c|c|c|c|c|}
\hline \multirow{2}{*}{ Año } & \multicolumn{2}{|c|}{ Pinus radiata } & \multicolumn{2}{|c|}{ Eucalyptus spp } & \multicolumn{2}{|c|}{ Otras } & \multicolumn{2}{|c|}{ Total } \\
\hline & ( ha ) & $(\%)$ & ( ha ) & $(\%)$ & ( ha ) & $(\%)$ & (ha ) & $(\%)$ \\
\hline 2006 & 503 & 39,9 & 470 & 37,3 & 288 & 22,8 & 1.261 & 100 \\
\hline 2007 & 234 & 33,3 & 391 & 55,6 & 78 & 11,1 & 704 & 100 \\
\hline 2008 & 269 & 31,3 & 370 & 43,0 & 220 & 25,6 & 859 & 100 \\
\hline 2009 & 231 & 22,7 & 704 & 69,2 & 83 & 8,1 & 1.018 & 100 \\
\hline 2010 & 281 & 26,1 & 515 & 47,7 & 282 & 26,2 & 1.078 & 100 \\
\hline 2011 & 153 & 17,5 & 398 & 45,5 & 323 & 37,0 & 874 & 100 \\
\hline Total & 1.671 & 28,8 & 2.848 & 49,2 & 1.275 & 22,0 & 5.793 & 100 \\
\hline Promedio & 278 & & 475 & & 212 & & 966 & \\
\hline
\end{tabular}

\section{Región de Los Lagos}

\begin{tabular}{|c|c|c|c|c|c|c|c|c|}
\hline \multirow{2}{*}{ Año } & \multicolumn{2}{|c|}{ Pinus radiata } & \multicolumn{2}{|c|}{ Eucalyptus spp } & \multicolumn{2}{|c|}{ Otras } & \multicolumn{2}{|c|}{ Total } \\
\hline & (ha ) & $(\%)$ & ( ha ) & $(\%)$ & (ha ) & $(\%)$ & (ha ) & $(\%)$ \\
\hline 2006 & 326 & 31,0 & 472 & 44,8 & 256 & 24,3 & 1.054 & 100 \\
\hline 2007 & 283 & 24,4 & 828 & 71,5 & 47 & 4,1 & 1.158 & 100 \\
\hline 2008 & 377 & 39,3 & 520 & 54,3 & 61 & 6,4 & 959 & 100 \\
\hline 2009 & 134 & 14,5 & 785 & 85,0 & 5 & 0,5 & 924 & 100 \\
\hline 2010 & 103 & 17,0 & 452 & 74,8 & 50 & 8,2 & 604 & 100 \\
\hline 2011 & 113 & 18,5 & 456 & 74,7 & 42 & 6,8 & 610 & 100 \\
\hline Total & 1.336 & 25,2 & 3.513 & 66,2 & 460 & 8,7 & 5.310 & 100 \\
\hline Promedio & 223 & & 586 & & 77 & & 885 & \\
\hline
\end{tabular}

